\title{
Imidazole Trimer-Water Complexes in Superfluid Helium Nanodroplets: Water Stretching Modes
}

\author{
Ahreum Ahn, Seung Jun Lee, Seulki Lee, Ahreum Min, Yusic Kim, Hyeon Jin Jung, \\ Seong Min Hong, Joon-Hwa Lee, ${ }^{*}$ Myong Yong Choi, ${ }^{*}$ and Roger E. Miller ${ }^{\dagger}$ \\ Department of Chemistry and Research Institute of Natural Science, Gyeongsang National University, Jinju 660-701, Korea \\ *E-mail: joonhwa@gnu.ac.kr andmychoi@gnu.ac.kr \\ ${ }^{\dagger}$ Department of Chemistry, The University of North Carolina at Chapel Hill, Chapel Hill, North Carolina 27599, U.S.A. \\ Received February 11, 2011, Accepted February 22, 2011
}

Key Words : Superfluid helium nanodroplets, Imidazole trimer-water complexes, DFT calculations, Infrared spectroscopy

Imidazole (IM) is an important moiety, having a proton donor and acceptor, in nucleic acid bases, i.e., adenine and guanine, and plays an important role in the intermolecular interactions of biological processes so that many theoretical and experimental studies of IM and its clusters have been investigated. ${ }^{1-7}$ Previously, we have presented and characterized the high-resolution infrared laser spectra of IM, imidazole dimer (IMD) and two isomers of IM- and IMDwater complexes (IMW and IMDW) formed in helium nanodroplets. ${ }^{8,9}$ Recently, we have extended the study of imidazole clusters to imidazole trimer (IMT) reporting the vibrational spectra of IMT in the free NH spectral region and found out that the IM molecules tend to form linear structures due to their dipole-dipole interactions in helium nanodroplets. ${ }^{10}$ The unambiguous free $\mathrm{NH}$ band assignment was made by the experimental techniques, such as pick-up oven temperature dependence and vibrational transition moment angle (VTMA), and density functional theory (DFT) calculations.

In the present note, higher order complexes, IMT with one water molecule (IMTWs), formed in He nanodroplets are presented. To the best of our knowledge, there is no report on the spectroscopic studies of IMTWs systems due to experimental difficulties in obtaining well resolved spectra. In this study we have used advantage of using helium nanodroplets since the very weak interactions between the target molecules and the helium give rise to almost unperturbed environments from that of the gas phase. Furthermore, the temperature of the helium nanodroplets is extremely low, $0.37 \mathrm{~K}$, thus the spectral broadening is significantly reduced making the He droplets as an ideal matrix for infrared spectroscopy. ${ }^{11,12}$

Using the helium nanodroplet techniques for obtaining well resolved spectra, we were able to measure the angle between the transition and permanent dipole moments of the target molecules, called the vibrational transition moment angles (VTMAs), which turned out to be a very sensitive structural and unambiguous vibrational band assignment

Deceased. November 6, 2005. University of North Carolina at Chapel Hill tool. Furthermore, VTMAs can be also easily calculated and compared with the experimental values obtained in this study. The use of VTMAs for the specific tautomer and vibrational band assignments of small biomolecules is well described in our previous reports. ${ }^{8,9,11,13-17}$ In this note we present the first spectroscopic investigation on the IMTW systems applying the VTMA techniques in He nanodroplets.

Figure 1 shows the two lowest energy structures for IMTWs $\left(>\mathrm{NH} \cdots \mathrm{OH}_{2}\right.$ and $\left.>\mathrm{N}: \cdots \mathrm{H}-\mathrm{O}-\mathrm{H}\right)$, where IMT is acting as a proton acceptor and donor from the nitrogen atom of the IM ring in the two lowest energy isomers, IMTW1 and 2, respectively. The structures were optimized at the B3LYP/6-311++G(d,p) level of theory and the calculated vectors representing the directions of the permanent electric dipole moments (solid arrows) and the vibrational transition moments (empty arrows) are superimposed onto the clusters. The magnitudes of the various moments for IMTWs are given in Table 1. It is clear from the figure that the patterns of VTMAs for the two isomers are quite different, making them a useful tool for distinguishing these
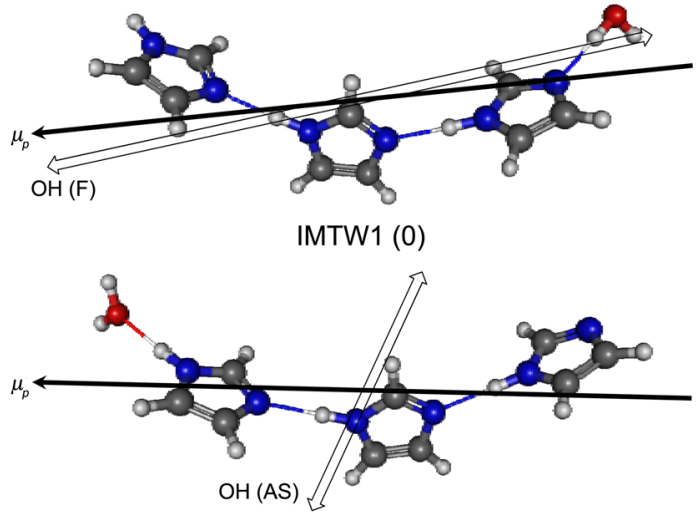

IMTW2 (2.3)

Figure 1. The two lowest energy isomers of imidazole trimer-water binary complexes (IMTW1 and IMTW2), showing the corresponding directions of the permanent electric dipole moments (solid arrows) and the vibrational transition moments (dashed double ended arrows) for the various vibrational modes. The values in the brackets give the energies relative to IMDW1 in $\mathrm{kJ} / \mathrm{mol}$ with a harmonic zero point energy correction. 
Table 1. A summary of the experimental frequencies and calculation data for the two lowest energy isomers of imidazole trimer-water complex (IMTW1 and IMTW2)

\begin{tabular}{cccccccc}
\hline $\begin{array}{c}\text { Imidazole water } \\
\text { clusters }\end{array}$ & $\begin{array}{c}\text { Exp. freq. } \\
\left(\mathrm{cm}^{-1}\right)\end{array}$ & $\begin{array}{c}\text { Harm. }{ }^{a} \text { freq. } \\
\left(\mathrm{cm}^{-1}\right)\end{array}$ & Assignment & $\begin{array}{c}\text { Calculated VTMAs } \\
(\text { degree })\end{array}$ & $\begin{array}{c}\text { DFT intensity } \\
(\mathrm{km} / \mathrm{mol})\end{array}$ & $\begin{array}{c}\text { Dipole moment } \\
(\text { Debye })\end{array}$ & $\begin{array}{c}\text { Relative }{ }^{b} \text { energy } \\
(\mathrm{kJ} / \mathrm{mol})\end{array}$ \\
\hline IMTW1 & 3715.6 & 3882.7 & $\mathrm{OH}(\mathrm{F})$ & 30 & 52 & 15.7 & 0 \\
IMTW2 & 3745.7 & 3918.5 & $\mathrm{H}_{2} \mathrm{O}(\mathrm{AS})$ & 72 & 101 & 17.2 & 2.3 \\
\hline
\end{tabular}

${ }^{a}$ The calculations were performed at the B3LYP/6-311++G(d,p) level. ${ }^{b}$ The energy was obtained with zero point energy correction.

two isomers and assigning the associated vibrational bands using the VTMA analysis, which will be described later.

The temperature dependence spectra of IM and its self associated clusters with water molecules in the region of $3710-3755 \mathrm{~cm}^{-1}$ were shown in Figure 2. The spectra were taken when the water vapor pressure was kept constant for the optimum pressure of one water molecule being picked up. The spectra (a), (b), and (c) were recorded at the pick-up IM oven temperatures of 39,48 , and $61{ }^{\circ} \mathrm{C}$, respectively. The bands near $3730 \mathrm{~cm}^{-1}$ are due to water monomer and high order water clusters, ${ }^{18,19}$ since those were obtained when the IM oven was absent and only the water source was open. No vibrational bands were observed in this spectral region when the water line was closed and only the IM oven was on. Therefore, the vibrational bands except for the bands marked with " $w$ " must then be attributed to the clusters of IM and their clusters with $\mathrm{H}_{2} \mathrm{O}$. Indeed, the bands labeled as "w" and " $\mathrm{w}_{2}$ " are due to the $\mathrm{P}(1)$ line of a single water molecule $\mathrm{e}^{18,19}$ and the $\mathrm{OH}$ stretching bands of $\mathrm{H}_{2} \mathrm{O}$ donor molecules in the water dimer, ${ }^{18-20}$ respectively. The band marked with " $\wedge$ " is due to water-nitrogen complexes from air-leak experiments. The bands labeled with "*” and "\#” were attributed to the IMW and IMDW clusters, respectively, derived from the IM oven temperature and water pick-up pressure dependence experiments. The band at higher and lower frequency was assigned to the asymmetric (AS) water stretching and free (F) $\mathrm{OH}$ mode, respectively., ${ }^{8,9}$ As the

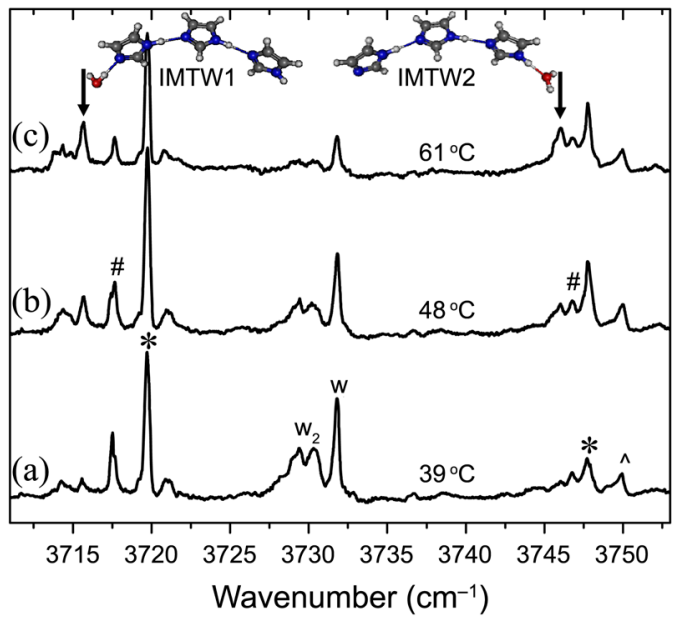

Figure 2. Temperature dependence spectra in the region of asymmetric water and free $\mathrm{OH}$ stretching modes for IMWs. Spectra (a), (b), and (c) correspond to the imidazole oven temperature of 39 , 48 , and $61{ }^{\circ} \mathrm{C}$, respectively. The bands marked with downwards arrows are attributed to IMTWs. The bands marked with "*” and "\#” are assigned to IMWs and IMDWs, respectively. pick-up IM oven temperature increased, from (a) to (c), the bands marked with downwards arrows kept growing, which means that a more probability of picking up higher order IM clusters at higher oven temperatures is likely, suggesting that the bands are due to the IMTW complexes.

To further confirm the band assignments of IMTWs, we have conducted the polarization dependence experiments in the spectral region of $3715-3750 \mathrm{~cm}^{-1}$, of which spectra are shown in Figure 3. Spectra (a), (b), and (c) in this region are obtained with a large direct current (DC) electric field $(80 \mathrm{kV} / \mathrm{cm})$ parallel, zero electric field, and perpendicular, respectively, to the permanent dipole moment of the target molecules, oriented in the laboratory frame of reference. When the applied field orients the dipole moment of a molecule, the excitation efficiency depends upon the direction and magnitude of both the vibrational transition moment and the laser electric field. As a result, the angle between the vibrational transition moment and the permanent electric dipole moment (VTMA) could be obtained by measuring the polarization angle that maximizes the band intensity. If the VTMA is less than the magic angle $\left(54.7^{\circ}\right)$, the

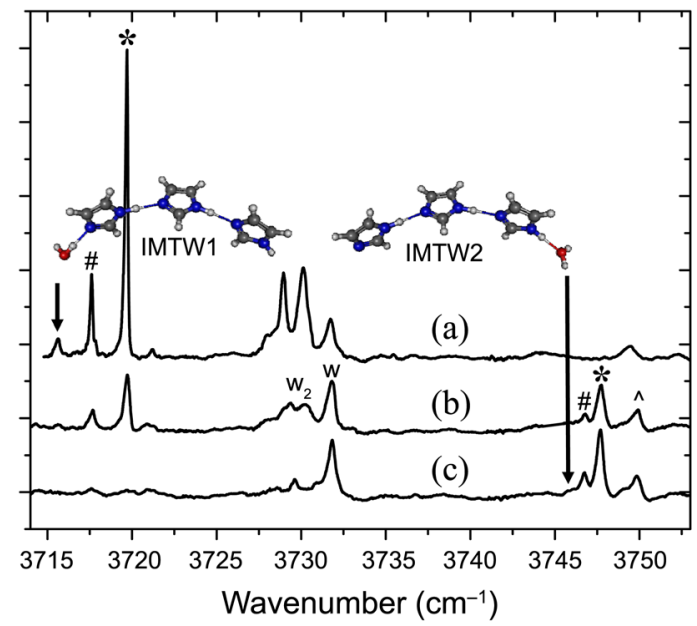

Figure 3. Polarization dependence spectra of the imidazole-water complexes. Infrared spectra (a), (b) and (c) correspond to parallel polarization, zero field and perpendicular polarization, respectively. The bands at 3745.7 and $3715.6 \mathrm{~cm}^{-1}$ marked with downwards arrows are due to the asymmetric water (IMTW2) and free (IMTW1) $\mathrm{OH}$ stretching mode of IMDWs, respectively, determined by the IM oven temperature and water pressure dependence experiments. The bands marked with "*" and "\#" are assigned to IMWs and IMDWs, respectively. The band marked with "^" is due to waternitrogen complexes. The bands near $3730 \mathrm{~cm}^{-1}$ marked with " $w$ " and " $\mathrm{w}_{2}$ " are related to the $\mathrm{P}(1)$ line of water monomer and the $\mathrm{OH}$ stretching band of $\mathrm{H}_{2} \mathrm{O}$ donor molecules in the water dimer, respectively. 


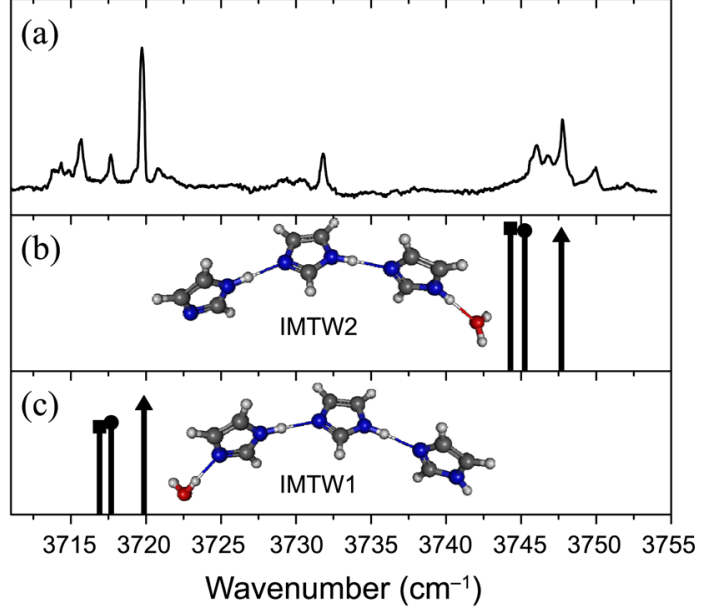

Figure 4. Comparison between the experimental spectrum taken at the IM oven temperature of $61{ }^{\circ} \mathrm{C}$ (a) and the DFT frequency calculations for IMTW2 (b) and IMTW1(a), scaled by a factor of 0.95554 and 0.9573 for the asymmetric water stretch and free $\mathrm{OH}$ mode, respectively. The calculations were conducted at the B3LYP/6-311++G(d,p) level of theory.

vibrational band associated with a parallel polarization, called a parallel band, is enhanced compared to the band from the zero field polarization and vice versa for a perpendicular band. ${ }^{8,9,11,13-17}$ Unfortunately, the spectra in Figure 3 were taken at a temperature of $36{ }^{\circ} \mathrm{C}$, which is much lower than the optimum temperature for IMT, so that no bands below the bands of IMDWs (3746.7 and 3717.6 $\mathrm{cm}^{-1}$ ) were observed, shown in Figure 3(b). However, the two bands appearing at 3715.6 and $3745.7 \mathrm{~cm}^{-1}$ are noticeable at parallel and perpendicular polarization scan, suggesting that the former is a parallel band while the latter is a perpendicular band. Even though the precise experimental VTMA analysis was not achieved due to the fairly low signal-to-noise ratios of the two bands at the lower IM pickup oven temperature $\left(36^{\circ} \mathrm{C}\right)$ for IMT, it is still evident that the calculated VTMAs of the two bands are very different each other, as shown in Figure 1 and Table 1, being 30 and $72^{\circ}$ for the free $\mathrm{OH}$ stretch of IMTW1 and the asymmetric water stretch of IMTW2, respectively. Therefore, the VTMA analysis of the two bands is also in good agreement between theory and experiments.

We now proceed to discuss the frequency gaps not only between the two bands of IMTWs, but also among the imidazole-water clusters, IMWs, IMDWs, and IMTWs. Figure 4(b) and (c) show the DFT bar spectra for the asymmetric water stretching mode of each imidazole-water clusters, i.e. IMW2 (triangle), IMDW2 (circle), IMTW2 (square) and the free $\mathrm{OH}$ stretching bands of the corresponding clusters of 1 's, respectively. We used a single scaling factor for the each vibrational mode to match for the asymmetric water stretch $(0.95554)$ and free $\mathrm{OH}$ mode $(0.9573)$ of IMW, IMDW, and IMTW.

The frequency gap between the two bands of IMTWs is $35.8 \mathrm{~cm}^{-1}$ without scaling factor corrections, which is in good agreement with the experimental value, $30.1 \mathrm{~cm}^{-1}$.

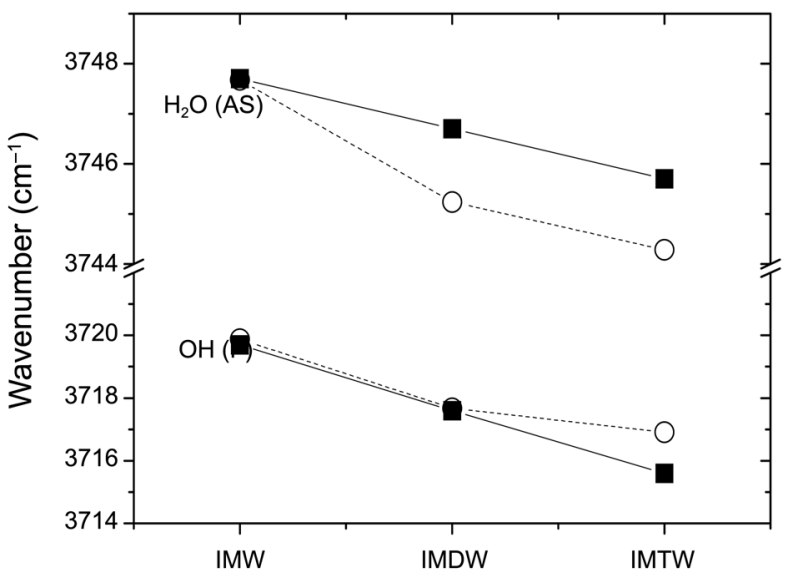

Figure 5. Frequency red-shift evolutions for the asymmetric stretch water (AS) and free (F) $\mathrm{OH}$ modes as a function of the size of imidazole-water clusters. Each experimental vibrational frequency (solid square) is compared with the predicted value (open circle) calculated from B3LYP/6-311++G** level of theory for the corresponding modes.

However, it is interesting to note that the calculated frequency gaps among the imidazole water clusters (IMWs, IMDWs, and IMTWs) for the corresponding bands are somewhat different from the experimental values. Figure 5 shows the frequency red-shift evolutions for the asymmetric water and free $\mathrm{OH}$ stretching modes as the size of imidazolewater clusters increases. Each experimental vibrational frequency (solid square) is compared with the predicted value (open circle) calculated from B3LYP/6-311++G** level of theory for the corresponding modes. The experimental frequency gap for the asymmetric water stretching band between IMTW/IMDW and IMDW/IMW is 1.0 and 1.0 $\mathrm{cm}^{-1}$, while for the free $\mathrm{OH}$ stretching band is 1.9 and 2.0 $\mathrm{cm}^{-1}$, showing a linear frequency shift as the IM clusters grow. However, calculated frequency gaps for the corresponding modes are 2.5 and $0.9 \mathrm{~cm}^{-1}$ for the asymmetric water stretching bands and 2.2 and $0.8 \mathrm{~cm}^{-1}$ for the free $\mathrm{OH}$ bands, showing a nonlinear frequency red-shift. Although we focus on the small frequency shift from the free stretching modes in the present study, it would be much interesting to investigate the sizeable red-shifts from the bonded $\mathrm{OH}$ and $\mathrm{NH}$ modes that are formed by hydrogen bonds between water and imidazole clusters in the near future. ${ }^{21}$

In summary, we report the first infrared laser spectra of the two isomers of imidazole trimer-water complexes (IMTWs) isolated in He nanodroplets in the region of $3710-3755 \mathrm{~cm}^{-1}$. The two bands at 3715.6 and $3745.7 \mathrm{~cm}^{-1}$ are assigned to the free $\mathrm{OH}$ and asymmetric water stretching mode of IMTW1 and 2, respectively, by using the IM oven temperature and water pressure dependence experiments. More conclusive evidence for the assignments was accomplished by the polarization dependence experiments. By orienting the molecular transition moments in the laboratory frame of reference with a large DC electric field, we have been able to enhance the parallel and perpendicular bands, associated to the free $\mathrm{OH}$ and asymmetric water stretching mode of 
IMTWs, respectively. The experimental observation of the two bands was well matched with the results of calculated vibrational transition moment angle (VTMA) of which values being one for parallel $\left(30^{\circ}\right)$ and the other for perpendicular $\left(72^{\circ}\right)$. For the weakly perturbed water stretching modes, we have found a linear frequency red-shift, i.e. 1 and $2 \mathrm{~cm}^{-1}$ redshifts of asymmetric and free $\mathrm{OH}$ stretching mode, respectively, in the modes as the imidazole clusters become larger from IMWs to IMTWs, while the theoretical calculations showed a non-linear red-shift. Further analysis of the linear frequency red-shifts of imidazole-water clusters in the bonded $\mathrm{OH}$ and $\mathrm{NH}$ spectral region will be reported. ${ }^{21}$

\section{Experimental Section}

Helium droplet beam apparatus used in this work was previously described in detail elsewhere, ${ }^{11,22}$ only a brief description is given here. The helium droplets are produced by a supersonic expansion of highly pure (99.9999\%) He gas through a $5 \mu \mathrm{m}$ diameter nozzle at a backing pressure of $50 \mathrm{~atm}$ and a nozzle temperature of $20 \mathrm{~K}$. Using the lognormal distribution of droplet sizes from the scaling laws, ${ }^{22}$ the mean size of He droplets are approximately $3000 \mathrm{He}$ atoms. The droplet beams pass by and pick up the target molecules at the exit of an effusive IM oven. A second pickup cell for water molecules is positioned downstream from the IM oven. Further downstream from the pick-up cells, a multi-pass/Stark cell ${ }^{22}$ is used to generate many crossings between the He droplet beams and the infrared laser. Furthermore, a large DC electric field is also applied to the interaction region for orienting the target molecules in the laboratory frame of reference. The infrared laser produced from a periodically poled lithium niobate (PPLN) cw-OPO having a stable output power of $70 \mathrm{~mW}$ in the spectral region of interest is guided into the multi-pass cell. The optical path is purged with dry $\mathrm{N}_{2}$ to take care of the laser power loss at the water absorption regions. The obtained spectra were calibrated using several external etalons and a wavemeter.

Acknowledgments. This work is supported by the Korea
Research Foundation (KRF) grant funded by the Korean Government (MEST) (No. 2009-0074609 and 2010-0005493) and (No. R01-2008-000-20002-0(2008)), in which main calculations were performed by using the supercomputing resource of the Korea Institute of Science and Technology Information (KISTI).

\section{References}

1. Fritscher, J. Phys. Chem. Chem. Phys. 2004, 6, 4950.

2. Yan, S.; Bu, Y. J. Phys. Chem. B 2004, 108, 13874.

3. Tatara, W.; Wojcik, M. J.; Lindgren, J.; Probst, M. J. Phys. Chem. A 2003, 107, 7827.

4. Poterya, V.; Profant, V.; Farnik, M.; Sistik, L.; Slavicek, P.; Buck, U. J. Phys. Chem. A 2009, 113, 14583.

5. Adesokan, A. A.; Chaban, G. M.; Dopfer, O.; Gerber, R. B. J. Phys. Chem. A 2007, 111, 7374.

6. Van Bael, M. K.; Smets, J.; Schoone, K.; Houben, L.; McCarthy, W.; Adamowicz, L.; Nowak, M. J.; Maes, G. J. Phys. Chem. A 1997, 101, 2397.

7. King, S. T. J. Phys. Chem. 1970, 74, 2133.

8. Choi, M. Y.; Miller, R. E. J. Phys. Chem. A 2006, 110, 9344.

9. Choi, M. Y.; Miller, R. E. Chem. Phys. Lett. 2009, 477, 276.

10. Lee, S.; Lee, S. J.; Ahn, A.; Kim, Y.; Min, A.; Choi, M. Y.; Miller, R. E. Bull. Korean Chem. Soc. 2011, 32, 885.

11. Choi, M. Y.; Douberly, G. E.; Falconer, T. M.; Lewis, W. K.; Lindsay, C. M.; Merritt, J. M.; Stiles, P. L.; Miller, R. E. Int. Rev. Phys. Chem. 2006, 25, 15.

12. Toennies, J. P.; Vilesov, A. F. Angew. Chem. Int. Ed. 2004, 43, 2622.

13. Min, A.; Lee, S. J.; Choi, M. Y.; Miller, R. E. Bull. Korean Chem. Soc. 2009, 30, 3039.

14. Lee, S. J.; Choi, M. Y.; Miller, R. E. Chem. Phys. Lett. 2009, 475, 24.

15. Choi, M. Y.; Dong, F.; Han, S. W.; Miller, R. E. J. Phys. Chem. A 2008, 112, 7185 .

16. Choi, M. Y.; Miller, R. E. J. Am. Chem. Soc. 2006, 128, 7320.

17. Choi, M. Y.; Dong, F.; Miller, R. E. Phil. Trans. R. Soc. A 2005, 363, 393.

18. Lindsay, C. M.; Douberly, G. E.; Miller, R. E. J. Mol. Struct. 2006, $786,96$.

19. Kuyanov, K. E.; Slipchenko, M. N.; Vilesov, A. F. Chem. Phys. Lett. 2006, 427, 5.

20. Frochtenicht, R.; Kaloudis, M.; Koch, M.; Huisken, F. J. Chem. Phys. 1996, 105, 6128.

21. Choi, M. Y.; Miller, R. E. In Preparation 2011.

22. Nauta, K.; Miller, R. E. J. Chem. Phys. 1999, 111, 3426. 